\title{
Effect of DNA Extraction Methods on the Apparent Structure of Yak Rumen Microbial Communities as Revealed by 16S rDNA Sequencing
}

\author{
YA-BING CHEN ${ }^{1}$, DAO-LIANG LAN ${ }^{2 *}$, CHENG TANG ${ }^{1}, \mathrm{XIAO}-N O N G ~ Y A N G^{1}$ and JIAN LI² \\ ${ }^{1}$ College of Life Science and Technology, Southwest University for Nationalities, Chengdu, Sichuan, PR China \\ ${ }^{2}$ Institute of Qinghai-Tibetan Plateau, Southwest University for Nationalities, Chengdu, Sichuan, PR China
}

Submitted 27 July 2014, reviewed 27 November 2014, accepted 27 November 2014

\begin{abstract}
To more efficiently identify the microbial community of the yak rumen, the standardization of DNA extraction is key to ensure fidelity while studying environmental microbial communities. In this study, we systematically compared the efficiency of several extraction methods based on DNA yield, purity, and 16S rDNA sequencing to determine the optimal DNA extraction methods whose DNA products reflect complete bacterial communities. The results indicate that method 6 (hexadecyltrimethylammomium bromide-lysozyme-physical lysis by bead beating) is recommended for the DNA isolation of the rumen microbial community due to its high yield, operational taxonomic unit, bacterial diversity, and excellent cell-breaking capability. The results also indicate that the bead-beating step is necessary to effectively break down the cell walls of all of the microbes, especially Gram-positive bacteria. Another aim of this study was to preliminarily analyze the bacterial community via $16 \mathrm{~S}$ rDNA sequencing. The microbial community spanned approximately 21 phyla, 35 classes, 75 families, and 112 genera. A comparative analysis showed some variations in the microbial community between yaks and cattle that may be attributed to diet and environmental differences. Interestingly, numerous uncultured or unclassified bacteria were found in yak rumen, suggesting that further research is required to determine the specific functional and ecological roles of these bacteria in yak rumen. In summary, the investigation of the optimal DNA extraction methods and the preliminary evaluation of the bacterial community composition of yak rumen support further identification of the specificity of the rumen microbial community in yak and the discovery of distinct gene resources.
\end{abstract}

Ke y w o r ds: $16 \mathrm{~S}$ rDNA sequencing, bacterial community, comparative analysis, DNA extraction methods, yak rumen

\section{Introduction}

As the first chamber of the ruminant stomach, the rumen contains abundant symbiotic microbes that perform important functions in the digestion of complex cellulolytic biomass and that supply nutrients to the host in the form of short-chain fatty acids and microbial proteins. Rumen microbes have been recently extensively studied due to their association with environmentally and economically important traits, such as feed conversion efficiency (Guan et al., 2008; Petri et al., 2013), methane production (Zhou et al., 2009), and the discovery of microbes or enzymes that facilitate the fermentation of biomass for biofuel production.

Rumen microbial community could be studied by culture-based or some molecular techniques (DGGE and ribosomal RNA clone libraries). Given the low sequencing depth of these approaches, previous community analyses represent a mere snapshot of the diverse community (Bibby et al., 2010; Fouts et al., 2012; Zhang etal., 2012). With the advent of next- generation sequencing technologies, conducting indepth sequencing on samples that are derived from any environment of choice, including the complex rumen microbial community at a deeper level than previously performed is feasible (Bergmann et al., 2010; Cuív et al., 2011; McOrist et al., 2002). Moreover, this technique is an effective tool for analyzing the bacterial community structure based on detailed taxonomic information. Notably, however, extracting DNA with a sufficient yield and high quality is a prerequisite to fully identify the members of the bacterial community with fidelity. Several studies reported that DNA extraction methods significantly impact the microbial community in samples from different habitats, including the rumen (Bergmann et al., 2010; Cuív et al., 2011; McOrist et al., 2002; Yu and Morrison 2004; Guo and Zhang, 2013). To enable a direct comparison of the rumen community structure from different individual samples, standardizing DNA extraction methods is crucial. Commercial DNA extraction kits for rumen content are currently unavailable, but kits that are designed for other samples

\footnotetext{
* Corresponding author: Dao-Liang Lan, Institute of Qinghai-Tibetan Plateau, Southwest University for Nationalities, Chengdu, Sichuan, PR China; e-mail: landaoliang@163.com
} 
are often used. Thus, the effectiveness of these cross-use kits should also be evaluated. Recently, several studies have evaluated DNA extraction methods for rumen content samples (Henderson et al., 2013; VillegasRivera et al., 2013). Here, we want to systematically compare the effectiveness of a variety of microbial DNA extraction methods for rumen based on the integrity, yield, purity, and sequencing results of extracted DNA.

Given the variation in the diet and inhabiting environment, different kinds of ruminants could harbor a distinct population of rumen microbes. Yak (Bos grunniens) lives exclusively on the Qinghai-Tibetan Plateau, China, at an average height of 3000 masl in a full-grazing style with coarse grasses as the only food due to poor living conditions (Qiu et al., 2012). As a result, special microbe species may exist in the yak rumen compared to those of other ruminants in plains. Moreover, with the development of modern breeding technologies and the abuse of antibiotics, the rumen microbial community has been subjected to change. Therefore, yaks in native areas (i.e., untouched by these modern advances) have a relatively complete and unbroken microbial community (An et al., 2005; Huang et al., 2012). Therefore, yaks can be considered an ideal animal model to study the microbial community and discover new microbes or valuable genes. For yaks, abundant humic substances and silt exist in the rumen, making DNA extraction difficult and maybe influencing subsequent sequencing. In the light of these aspects, this study aimed at selecting a method for DNA extraction from the yak rumen and preliminary analysis of the basic bacterial community structure in the yak rumen.

\section{Experimental}

\section{Materials and Methods}

Ethics statement and sample collection. Yaks were supplied by the Hongyuan Yak Breeding Farm (N32 $47^{\prime} 35.50^{\prime}$ latitude, E102 $32^{\prime} 34.32^{\prime}$ longitude, average 3600 masl), Sichuan, China. The experimental procedures and the collection of rumen samples that were used for this study were approved according to the animal care and use of laboratory animals of the Institutional Animal Care and Use Committee of the Southwest University for Nationalities. Samples of the rumen contents were collected immediately after death and transported in an airtight container full of dry ice to the laboratory within $30 \mathrm{~min}$. Then, the samples were stored at $-80^{\circ} \mathrm{C}$.

Acquisition of microbial pellets. One gram of rumen content from each yak was mixed to form a sample pool (total amount $3.0 \mathrm{~g}$ ). For DNA extraction, $2.0 \mathrm{~g}$ of rumen content was thawed on ice and transferred to a 50-ml tube. A 20-ml portion of PBS buffer was added to the tube to wash the rumen content, and the mixture was vortexed thoroughly for $2 \mathrm{~min}$. After centrifugation ( $2 \mathrm{~min}, 200 \mathrm{~g}$ ), the supernatant was collected. The samples were washed 3 times, and the total mixed supernatant was then centrifuged $(2 \mathrm{~min}, 12,000 \mathrm{~g})$ to obtain microbial pellets for DNA extraction.

Extraction of DNA from the rumen content. Based on the existing DNA extraction methods that were designed for other samples and on the characteristics of the yak rumen (Nettmann et al., 2008; Willner et al., 2012), we summarized and improved several extraction methods and further compared their DNA extraction efficiency. The specific parameters of every method are listed in Table I.

Table I

Parameters of 10 improved DNA extraction methods

\begin{tabular}{|l|c|c|c|c|c|c|c|c|c|c|}
\hline \multirow{2}{*}{ Parameter } & \multicolumn{10}{|c|}{ DNA extraction methods } \\
\cline { 2 - 12 } & 1 & 2 & 3 & 4 & 5 & 6 & 7 & 8 & 9 & 10 \\
\hline CTAB $^{\text {a }}$ & + & + & + & + & + & + & - & - & - & - \\
\hline SDS $^{\mathrm{b}}$ & + & + & + & + & + & + & + & + & + & + \\
\hline Proteinase K $^{\mathrm{c}}$ & + & + & + & + & + & + & + & + & + & + \\
\hline Lysozyme & + & + & + & - & - & - & + & + & + & - \\
\hline Freeze-thaw & - & + & - & - & + & - & - & + & - & - \\
\hline Bead beating & - & - & + & - & - & + & - & - & + & - \\
\hline Silica column & - & - & - & - & - & - & - & - & - & + \\
\hline
\end{tabular}

a: Treatment with $5 \%$ CTAB (w/v).

b: Treatment with $1 \% \operatorname{SDS}(\mathrm{w} / \mathrm{v})$.

c: Treatment with $0.3 \mathrm{mg} / \mathrm{ml}$ proteinase $\mathrm{K}$.

d: Treatment with $0.3 \mathrm{mg} / \mathrm{ml}$ lysozyme.

Method 1 (CTAB-SDS-Lysozyme):Total genomic DNA was isolated by the method of Nettmann, et al. (2008) which includes an enzymatic cell lysis with lysozyme and two chemical lysis with SDS and CTAB as detergent, respectively. The DNA was purified with chloroform-isoamyl alcohol, precipitated with isopropanol and dissolved in $30 \mu \mathrm{l}$ of nuclease-free water.

Method 2 (CTAB-SDS-Lysozyme- physical lysis by freeze-thaw): According to the protocol 1, there exists some modification: after the CTAB solution was added to cell suspension, three cycles of freezing in $-80^{\circ} \mathrm{C}$ refrigerator for $10 \mathrm{~min}$. and heating in a water bath at $65^{\circ} \mathrm{C}$ was performed until the samples was completely thawed.

Method 3 (CTAB-SDS-Lysozyme- physical lysis by bead-beating): Referring to method 1, there are some changes in method 3 , after treating the cell suspension with lysozyme $(0.3 \mathrm{mg} / \mathrm{mL}), 100 \mathrm{mg}$ of zirconium-silica beads $(0.1 \mathrm{~mm}$ diameter) was added to the mixture. Next, the mixture was vortexed thoroughly for $10 \mathrm{~min}$.

Method 4 (CTAB-Lysozyme): For chemical cell lysis by CTAB and enzymatic lysis by lysozyme, the DNA isolation protocol of Nettmann, et al. (2008) was used. 
Method 5 (CTAB-Lysozyme- physical lysis by freezethaw): The protocol combined the chemical enzymatic cell lysis and physical lysis by freeze-thaw, referring to methods 2 and 4 , the specific process was designed.

Method 6 (CTAB-Lysozyme- physical lysis by beadbeating): The protocol combined the chemical enzymatic cell lysis and physical lysis by beads, referring to methods 3 and 4, the specific process was designed.

Method 7 (SDS-lysozyme): Firstly, the cell pellet was lysed with lysozyme $(0.3 \mathrm{mg} / \mathrm{mL})$, the samples were incubated at $37^{\circ} \mathrm{C}$ for $60 \mathrm{~min}$., followed by an chemical cell lysis using SDS an detergent.

Method 8 (SDS-lysozyme- physical lysis by freezethaw): For protocol 8, the DNA was obtained by combined freeze-thaw lysis and chemical cell lysis (refer to methods 2 and 7).

Method 9 (SDS-lysozyme-physical lysis by beadbeating): For protocol 9, the DNA was obtained by combined bead-beating lysis and chemical cell lysis (refer to methods 3 and 7).

Method 10 (DNA Extraction Kit): The procedure was performed according to the instructions of QIAamp DNA Stool Extraction Kit (Qiagen, Germany).

Determination of the DNA yield, purity, and fragment distribution. The DNA yields of the different methods were calculated using the NanoDrop ND-3300 spectrometer. The purity of the extracted DNA was assessed via the values of OD260/OD280 and OD260/ OD230. The size of the isolated DNA fragments was determined by agarose gel electrophoresis using the $\lambda$-Hind III Marker as a reference.

PCR amplification for pyrosequencing. The V3 to $\mathrm{V} 4$ regions of $16 \mathrm{~S}$ rDNA genes from bacteria were amplified using the bar-coded primer set of $338 \mathrm{~F}$ (5'ACTCCTACGGGAGGCAGCA-3') and 806R (5'GGACTACHVGGGTWTCTAAT-3'). The PCR products were examined on an agarose gel, and the DNA bands were excised and purified. In addition, the concentration and qualities of the DNA were determined using the TBS-380 fluorophotometer. DNA pool was prepared by mixing together equal quantities of DNA samples of three DNA samples per treatment and then sent to BGI (Shenzhen, China) for sequencing on the Illumina MiSeq PE300 platform (Illumina, USA).

Sequence processing. Prior to taxonomic analysis, the DNA sequences were subjected to the following filtering steps: (1) sequences containing windows of 50 consecutive base pairs with an average quality score of less than 20 were truncated at the start of the low-quality region; (2) pair-end reads were combined into one sequence, and the length of overlap was longer than $10 \mathrm{bp}$; and (3) the mismatch rate for the overlap region of assembled sequences was less than 0.2 ; otherwise, the sequences were removed. Furthermore, those sequences with a $97 \%$ identity cutoff were labeled as an operational taxonomic unit (OTU). After the above filtering, RDP (Ribosomal Database Project) Classifier was used to assign the sequences to different taxonomy levels at a bootstrap cutoff of 30\%. The pyrosequencing reads were then aligned using Infernal based on the bacterial alignment model in the Align module of RDP. By applying Complete Linkage Clustering, the sequences in each sample were assigned to phylotype clusters of $97 \%$ identity. Based on these clusters, a Rarefaction curve (Colwell and Coddington, 1994; Schloss and Handelsman, 2005), ACE richness estimations, and Shannon diversities (Gotelli, 2002) were generated using RDP software.

\section{Results}

Extraction efficiency, purity, and integrity. The length of all of the fragments appeared to be at about $20 \mathrm{~kb}$. The yield and the OD260/OD280 ratio are shown in Table II. Methods 3 and 6, which included a bead-beating step, produced highest yield and purest DNA compared to the other protocols, suggesting that bead beating is necessary in rumen DNA extraction. Methods 7, 8, and 9, which did not use CTAB (hexadecyltrimethylammomium bromide) as a detergent to disrupt the cell and yielded low DNA, demonstrating that the chemical lysis of CTAB is also required for DNA extraction from rumen content samples.

Table II

Yield and purity of DNA extracted using 10 methods

\begin{tabular}{|c|c|c|}
\hline Sample & Yield $(\mathrm{ng} / \mu \mathrm{l})$ & OD260/280 \\
\hline 1 & $336.61 \pm 7.22$ & $1.60 \pm 0.08$ \\
\hline 2 & $455.30 \pm 6.55$ & $1.53 \pm 0.12$ \\
\hline 3 & $527.48 \pm 5.62$ & $1.85 \pm 0.13$ \\
\hline 4 & $324.12 \pm 6.88$ & $1.72 \pm 0.12$ \\
\hline 5 & $384.81 \pm 7.82$ & $1.79 \pm 0.21$ \\
\hline 6 & $525.55 \pm 11.22$ & $1.88 \pm 0.11$ \\
\hline 7 & $59.71 \pm 12.11$ & $1.82 \pm 0.21$ \\
\hline 8 & $39.03 \pm 0.23$ & $1.65 \pm 0.12$ \\
\hline 9 & $49.64 \pm 0.75$ & $1.81 \pm 0.15$ \\
\hline 10 & $62.05 \pm 0.24$ & $1.81 \pm 0.15$ \\
\hline
\end{tabular}

Sequencing results. Considering the requirements of DNA quantity and quality for sequencing, DNA that was extracted using Methods 2 and 8 could not be successfully analyzed. A total of 191,349 raw sequences from all of the samples were generated. After trimming, sorting, and quality control, 171,231 or $89.5 \%$ of the sequences were used in our analysis. Specific sequence information for each method is included in Table III. Consistent with the quantitative results, the number of 
Table III

Specific sequence information of each method

\begin{tabular}{|c|c|c|c|}
\hline Sample & Sequences & Bases (bp) & Average Length (bp) \\
\hline 1 & 25465 & 10022673 & 393.59 \\
\hline 3 & 26080 & 10270236 & 393.8 \\
\hline 4 & 23216 & 9134524 & 393.46 \\
\hline 5 & 20325 & 7997392 & 393.48 \\
\hline 6 & 29763 & 11722005 & 393.84 \\
\hline 7 & 10446 & 4114615 & 393.89 \\
\hline 9 & 15781 & 6211301 & 393.59 \\
\hline 10 & 20155 & 7944212 & 394.16 \\
\hline
\end{tabular}

Table IV

Specific OTUs and OTU-based diversity index of different extraction treatments

\begin{tabular}{|c|r|r|c|}
\hline \multirow{2}{*}{ Sample } & \multicolumn{3}{|c|}{ Diversity index (0.97) } \\
\cline { 2 - 4 } & OTU & Ace & Shannon \\
\hline 1 & 937 & 1069 & 5.24 \\
\hline 3 & 942 & 1093 & 5.24 \\
\hline 4 & 953 & 1084 & 5.22 \\
\hline 5 & 929 & 1055 & 5.4 \\
\hline 6 & 1026 & 1126 & 5.46 \\
\hline 7 & 824 & 983 & 5.47 \\
\hline 9 & 914 & 1055 & 5.44 \\
\hline 10 & 829 & 898 & 5.48 \\
\hline
\end{tabular}

raw sequences of methods 3 and 6 were also larger than those of other methods.

OUT-based analysis. Fig. 1 displays the rarefaction plots for each sample, in which at least 800 OTUs were observed, indicating that the rumen microbial community is complex. Table IV lists the number of OTUs and

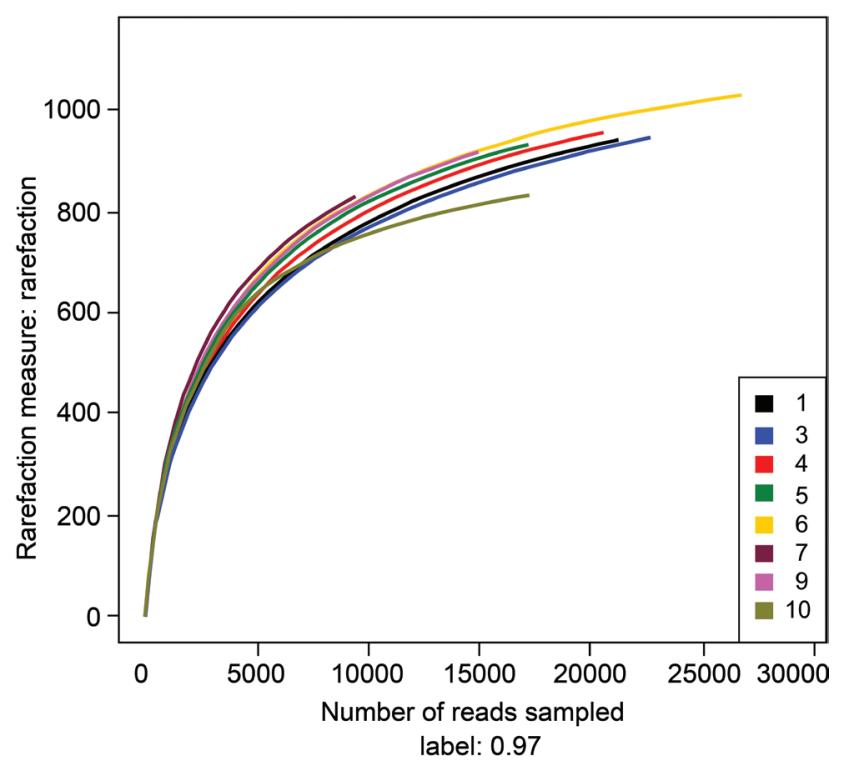

Fig. 1. Rarefaction curves of the rumen microbial community based on 16S rRNA gene sequencing (at the 0.97 identity level). the diversity index of each DNA extraction treatment. The ACE index was usually used to calculate the richness of the output, and the Shannon index could reflect the diversity of microorganisms. A larger value of Shannon indicates more-abundant species in a sample. The treatments with the bead-beating step had evidently higher numbers of OTUs and a higher diversity index than the others, suggesting that robust bead beating is necessary for cell lysis. In contrast, the QIAamp kit had fewer OTUs but a higher diversity index, indicating that this kit was efficient in cell lysis but lost much DNA during the subsequent purification steps.

Abundant genera in each sample were selected and compared via their abundance in other samples, as shown in Fig. 2. In the heat map, the lower number of the blue blocks (indicating low abundance) in the total OTUs and the greater abundance of dominant genera translate into a more efficient method for DNA extraction from different kinds of microorganisms. Therefore, we speculated that methods 6 and 9 were more representative than other methods. In addition, a cluster analysis indicated that methods 7,8 , and 9 clustered together and other traditional methods were included in another cluster, suggesting that bead beating was determinative in DNA extraction.

Comparison of Gram-positive bacterial abundance. As shown in Fig. 3., the abundances of the top eight Gram-positive genera in each sample were investigated to further compare the efficiencies of cell lysis for different treatments. Among these methods, method 6 exhibited the best capability for cell lysis; methods 1 and 3 also performed well. Moreover, for three Gram-positive genera with lower abundances (Ruminococcus, Acetitomaculum, and Mogibacterium), the treatments with bead beating evidently had a higher abundance than did the other methods. This observation indicates that robust mechanical homogenization is required to efficiently disrupt Gram-positive bacteria. When the cells were lysed gently, certain rare Gramnegative phyla, including Fibrobacteres, Proteobacteria, and Tenericutes, were actually overestimated.

Taxonomy-based analysis of rumen microbes. Based on the above results, method 6 was deemed as effective for extracting complete DNA from all of the microbes. A taxonomy-based analysis of sample 6 showed that the rumen bacterial community consisted of 21 phyla, 35 classes, 52 orders, 75 families, and 112 genera. At the phylum level, the community structure is shown in Fig. 4. Within the bacterial population, 21 phyla were found across the rumen contents. The bacterial composition of the rumen consisted mainly of the Firmicutes and Bacteroidetes phyla, at $64 \%$ and $20 \%$ of the total reads on average, respectively. The remaining microbes consisted of low-abundance phyla $(<10 \%$ of the total reads), such as Fibrobacter, Spirochaeta, and 


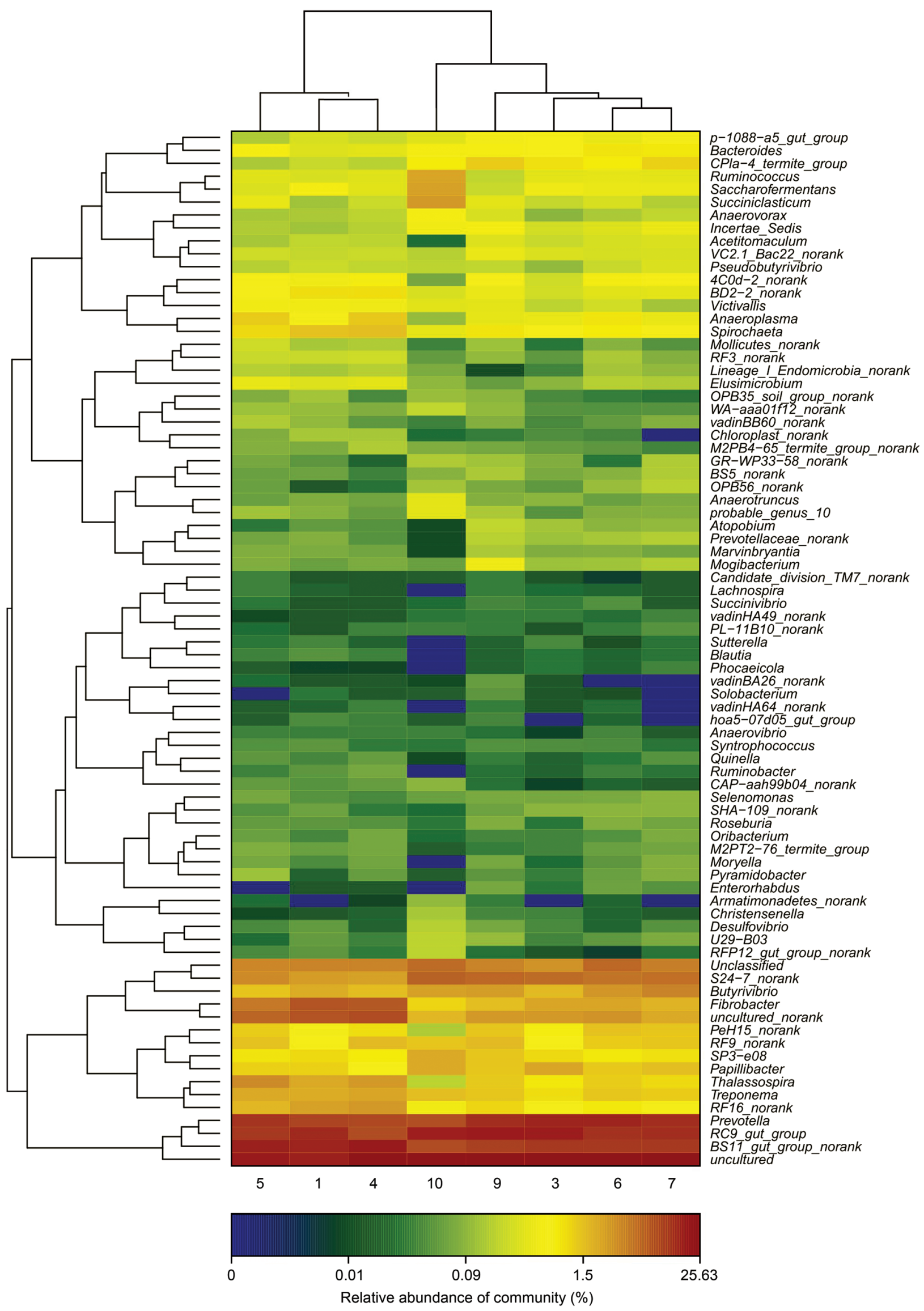

Fig. 2. Heat map analysis of the bacterial community based on the top 50 genera. The top 50 most-abundant genera in each sample were selected (a total of 80 genera for all 8 treatments) and compared with their abundance in other samples. The color intensity in each panel shows the percentage of a genus in a sample, referring to the color key at the bottom. 


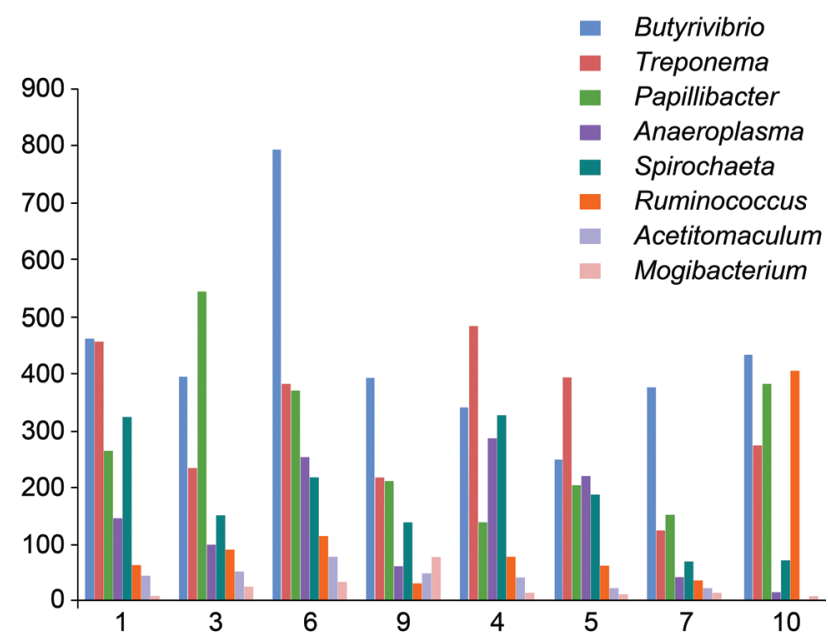

Fig. 3. The abundance of the top eight Gram-positive genera in the different treatments.

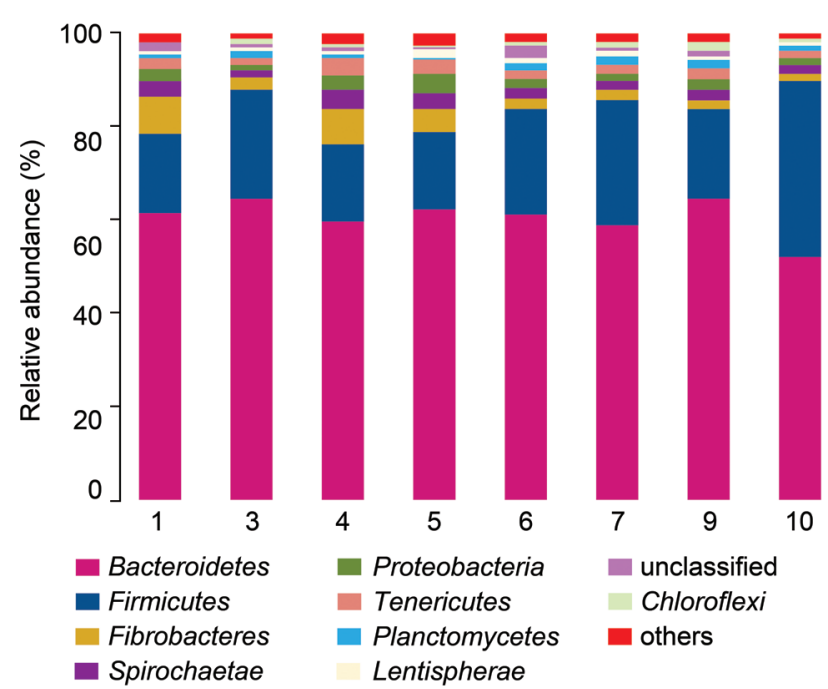

Fig. 4. Microbial community structures of the samples of yak rumen. The relative abundance of bacterial 16S rRNA genes was estimated through classification at the phylum level.

Proteobacteria, which represented approximately $1.7 \%$, $2.3 \%$, and $1.8 \%$, respectively. Notably, the five mostabundant phyla in the yak rumen accounted for $90 \%$ of the analyzed sequences, and the phylum distribution was similar to those of other ruminants. At the genus level, Prevotella, Butyrivibrio, Fibrobacter, the uncultured, and two poorly described genera (RC9_gut_group and BS11_gut_group_norank) were identified as the dominant genera in the rumen bacterial community.

\section{Discussion}

Comparison of the DNA extraction methods. With the advent of next-generation sequencing technologies, conducting in-depth sequencing and data analysis on the rumen microbial community and the exploration of uncultured microorganisms are feasible. However, effective DNA extraction with high quality is the premise. An increasing number of reports exist regarding the biases of different methods during DNA extraction as revealed by $16 \mathrm{~S}$ rDNA sequencing. However, information about the yak rumen content is scarce. Compared to other ruminants in plains, yaks live in a free-ranging pattern; therefore, the yak rumen usually contains much silt and many humic substances that complicate DNA extraction. The aim of the current study was to select one optimal method to extract DNA from yak rumen microbes as evaluated by $16 \mathrm{~S}$ rDNA sequencing. The factor that primarily affects DNA yield is the efficiency of cell lysis. The methods with bead beating produced more DNA than did the other methods, indicating that bead-beating is efficient for cell lysis. Method 9, which included the bead-beating step, produced less DNA, suggesting the importance of $\mathrm{CTAB}$ in cell lysis.

For PCR-based community analysis, the quantity of DNA is not the key factor because trace DNA (>500 ng) is adequate for PCR amplification and subsequent sequencing. As shown in our results, both protocols 7 and 9 produced DNA of low quantity and quality, but the OTU-based analysis generated an almost fair profile of the bacterial community. Interestingly, method 2 produced a relatively higher amount of DNA. However, the DNA did not meet the requirements for $16 \mathrm{~S} \mathrm{rDNA}$ sequencing This exclusion may be due to numerous DNA fragments with small segment sizes as a result of robust shear force during the freeze-thaw and chemical cleavage processes, prohibiting the DNA from serving as a template for PCR amplification. Moreover, the PCR reaction would also be affected if certain impurities (i.e., humic acid) existed in the DNA content. For sample 8 , the DNA quantity did not meet the further analysis requirements, resulting in analytical failure.

The total OTU number and the relevant diversity indexes can be used to evaluate the efficiencies of the DNA extraction methods. In general, more OTUs and higher diversity indexes may represent more species within samples. Therefore, the methods with beadbeating steps are significantly better than are other methods that use chemical lysis or freeze-thaw. The kit effectively lysed the cell walls of bacteria but lost much DNA during following steps, resulting in larger diversity index but fewer OTUs. Due to the thick cell well and spore formation, the Gram-positive bacteria are relatively resistant to both detergents and mechanical lysis. Therefore, we believe that more Gram-positive bacteria detected indicated more-efficient DNA extraction methods. For ruminants, Firmicutes is a relatively dominant phylum in the rumen, performing essential functions in energy conversion. Actinobacteria is also an important Gram-positive bacterium that regulates 
polymer degradation, glycogen accumulation, and polyphosphate accumulation. In terms of these two phyla, method 6 was the most efficient because more Gram-positive bacteria were detected.

Microbial community of yak rumen. Given the significant differences in their diet and habitats, yaks could harbor a distinct population of rumen bacteria compared to that of other ruminants. Currently, the rumen microbes of yak have not received adequate attention. Therefore, another aim of the present study was to preliminarily analyze the bacterial community via 16S rRNA high-throughput sequencing. Previously, An et al. (2005) compared the prokaryote diversity in the rumen between yak and cattle as estimated by $16 \mathrm{~S}$ rDNA clone library analysis. Given the limitations of the sequencing depth, An et al. sequenced fewer fragments than in their study (An et al., 2005). 16S rDNA sequencing is an efficient tool to fully explore valuable gene resources and to understand the specificity of the bacterial community from any sample, including yak rumen content. Huang et al. (2012) compared the methanogen diversity from "energy-saving" yak and cattle based on $16 \mathrm{~S}$ rDNA sequencing to explain why yaks produce less methane than do cattle. This previous study revealed that the methanogen community structure of yak was significantly different from that of cattle, ultimately resulting in less methane production and indicating the effectiveness of the technique in analyzing the microbial community. Unfortunately, the results cannot truly reflect the microbial community due to the limited capacity of the QIAamp DNA Stool Kit for rumen DNA extraction. In this study, the $16 \mathrm{~S}$ rDNA sequencing results demonstrated that the bacterial community of the yak rumen included approximately 21 phyla, 35 classes, 75 families, and 112 genera. Compared with cattle, the percentage of taxa in yak is significantly different in relative abundance, ranging from $10.5 \%$ at a phyla level to $105.5 \%$ at a genus level (Jami and Mizrahi 2012; Omoniyi et al., 2014; Ross et al., 2012). The sequencing results revealed that, at the phylum level, the microbial community of the yak rumen was dominated by Bacteroidetes and Firmicutes, and the microbial distribution of the major phyla is similar to that of cattle (Zened et al., 2013), thereby suggesting the importance of the two phyla in ruminal metabolism (Turnbaugh et al., 2008).

The sequencing results also indicate that uncultured or unclassified species in the yak rumen comprise a large proportion of the bacterial community. For example, RC9_gut_group and BS11_gut_group_norank accounted for a large percentage of the total bacteria (13.12\% and $10.10 \%$, respectively). Moreover, numerous rare, undescribed genera, such as CAP-aah99b04norank, SHA-109-norank, and M2PT2-76_termite_ group, were also detected in the present study. Similarly,
An et al. (2005) found that a higher ratio of uncultured microbial species was also identified by clone library analysis in the yak rumen content. We can speculate, therefore, these bacteria may possess important and yet unrecognized ecological functions and occupy a special ecological niche in the rumen.

Regarding ruminants, Fibrobacter and Ruminococcus are well-known fibrolytic species in rumen. Yaks are predicted to harbor more fibrolytic microorganisms in the rumen because their feed mainly consists of fibercontaining grasses. The results show that Fibrobacter in the rumen represent approximately $2.5 \%$ of all of the reads, which is similar to that of cattle. Interestingly, the percentage of Ruminococcus in yak $(0.4 \%)$ is significantly less than that in cattle (5\%) (Jami and Mizrahi, 2012), and the significant variance in the abundance is an important subject for further research. One explanation could be that other dominant fibrolytic microorganisms exist in yak.

The species of the genus Prevotella consist of a large group of bacteria with functional diversity, including promoting initial dietary protein breakdown and acting synergistically with cellulolytic species to improve the ruminal cellulolytic capacity. The genus Prevotella is the core bacterial genus, with approximately $40 \%$ to $50 \%$ percentage of all bacteria in adult dairy cattle or cattle. However, in the present study, the Prevotella in yak rumen only represents $15 \%$. The significant variation may be attributed to differences in the available nutrients; dairy cattle and cattle are fed with high-quality forage and grain, whereas yak mainly feed on coarse grasses. Several studies have found that the identified diet impacts the bacterial community. Petri et al. (2013) reported that the percent of Prevotella varied significantly based on the dietary composition and accounted for approximately $8.9 \%, 12.8 \%$, and $31.6 \%$ in cattle that were fed with forage, mixed forage, and high grain, respectively (Petri et al., 2013). In summary, the diet has a significant influence on the bacterial community in the yak rumen.

\section{Acknowledgements}

This work was supported by the Veterinary Medicine Discipline Program of the Southwest University for Nationalities (2014XWDS0906), Key Natural Science Project of Education Department in Sichan province (15ZB0486), and National Science \& technology Pillar Program of China (2012BAD13B06).

\section{Literature}

An D., X. Dong and Z. Dong. 2005. Prokaryote diversity in the rumen of yak (Bos grunniens) and Jinnan cattle (Bos taurus) estimated by $16 \mathrm{~S}$ rDNA homology analyses. Anaerobe. 11: 207-215.

Bergmann I., K. Mundt, M. Sontag, I. Baumstark, E. Nettmann and M. Klocke. 2010. Influence of DNA isolation on Q-PCR-based 
quantification of methanogenic Archaea in biogas fermenters. Syst. Appl. Microbiol. 33: 78-84.

Bibby K., E. Viau and J. Peccia. 2010. Pyrosequencing of the 16 S rRNA gene to reveal bacterial pathogen diversity in biosolids. Water. Res. 44: 4252-4260.

Colwell R.K. and J.A. Coddington. 1994. Estimating terrestrial biodiversity through extrapolation. Philos. Trans. R. Soc. Lond. B. Bio. Sci. 345: 101-118.

Cuív P.Ó., D.A. Cárcer, M. Jones, E.S. Klaassens, D.L. Worthley, V.L. Whitehall, S. Kang, C.S. McSweeney B.A. Leggett and M. Morrison. 2011. The effects from DNA extraction methods on the evaluation of microbial diversity associated with human colonic tissue. Microb. Ecol. 61: 353-362.

Fouts D.E., S. Szpakowski, J. Purushe, M. Torralba, R.C. Waterman, M.D. MacNeil, L.J. Alexander and K. E. Nelson. 2012. Next generation sequencing to define prokaryotic and fungal diversity in the bovine rumen. PLoS One. 7: e48289.

Gotelli N.J. 2002. Ecology: Biodiversity in the scales. Nature 419: 575-576.

Guan L.L., J.D. Nkrumah, J.A. Basarab and S.S. Moore. 2008. Linkage of microbial ecology to phenotype: correlation of rumen microbial ecology to cattle's feed efficiency. FEMS Microbiol. Lett. 288: 85-91.

Guo F. and T. Zhang. 2013. Biases during DNA extraction of activated sludge samples revealed by high throughput sequencing. Appl. Microbiol. Biotechnol. 97: 4607-4616.

Henderson G., F. Cox, S. Kittelmann, V.H. Miri, M. Zethof, S.J. Noel, G.C. Waghorn and P.H. Janssen. 2013. Effect of DNA Extraction methods and sampling techniques on the apparent structure of cow and sheep sumen microbial communities. PLoS One. 8: e74787.

Huang X.D., H.Y. Tan, R.J. Long, J.B. Liang and A.D. Wright. 2012. Comparison of methanogen diversity of yak (Bos grunniens) and cattle (Bos taurus) from the Qinghai-Tibetan plateau, China. BMC Microbiol. 12: 237.

Jami E. and I. Mizrahi. 2012. Composition and similarity of bovine rumen microbiota across individual animals. PLoS One. 7: e33306.

McOrist A.L., M. Jackson and A.R. Bird. 2002. A comparison of five methods for extraction of bacterial DNA from human faecal samples. J. Microbiol. Methods. 50: 131-139.

Nettmann E., I. Bergmann, K. Mundt, B. Linke and M. Klocke. 2008. Archaea diversity within a commercial biogas plant utilizing herbal biomass determined by $16 \mathrm{~S} \mathrm{rDNA}$ and mcrA analysis. J. Appl. Microbiol. 105: 1835-1850.
Omoniyi L.A., K.A. Jewell, O.A. Isah, A.P. Neumann, C.F. Onwuka, O.M. Onagbesan and Suen G. 2014. An analysis of the ruminal bacterial microbiota in West African Dwarf sheep fed grass and tree-based diets. J. Appl. Microbiol. 116: 1094-1105.

Petri R.M., T. Schwaiger, G.B. Penner, K.A. Beauchemin, R.J. Forster, J.J. McKinnon and T.A. McAllister. 2013. Characterization of the core rumen microbiome in cattle during transition from forage to concentrate as well as during and after an acidotic challenge. PLoS One. 8: e83424.

Qiu Q., G. Zhang, T. Ma, W. Qian, J.Wang, Z. Ye, C. Cao, Q. Hu, J. Kim and D.M. Larkin. 2012. The yak genome and adaptation to life at high altitude. Nature. Genet. 44: 946-949.

Ross E.M, P.J Moate, C.R. Bath, S.E. Davidson, T.I. Sawbridge, M. Guthridge, B.G. Cocks and B.J. Hayes. 2012. High throughput whole rumen metagenome profiling using untargeted massively parallel sequencing. BMC Genet. 13: 53.

Schloss P.D. and J. Handelsman. 2005. Introducing DOTUR, a computer program for defining operational taxonomic units and estimating species richness. Appl. Environ. Microbiol. 71: 1501-1506 Turnbaugh P. J., F. Bäckhed, L. Fulton and J.I. Gordon. 2008. Dietinduced obesity is linked to marked but reversible alterations in the mouse distal gut microbiome. Cell. Host. Microbe. 3: 213-223. Villegas-Rivera G., Y. Vargas-Cabrera, N. González-Silva, F. Aguilera-García, E. Gutiérrez-Vázquez, A. Bravo-Patiño, M. CajeroJuárez, V.M. Baizabal-Aguirre and J.J. Valdez-Alarcón. 2013. Evaluation of DNA extraction methods of rumen microbial populations. World. J. Microbiol. Biotechnol. 29: 301-307.

Willner D., J. Daly, D. Whiley, K. Grimwood, C.E. Wainwright and P. Hugenholtz. 2012. Comparison of DNA extraction methods for microbial community profiling with an application to pediatric bronchoalveolar lavage samples. PLoS One. 7: e34605.

Yu Z. and M. Morrison. 2004. Improved extraction of PCR-quality community DNA from digesta and fecal samples. Biotechniques. 36 : 808-813.

Zened A., S. Combes, L. Cauquil, J. Mariette, C. Klopp, O. Bouchez, A. Troegeler-Meynadier and F. Enjalbert. 2013. Microbial ecology of the rumen evaluated by 454 GS FLX pyrosequencing is affected by starch and oil supplementation of diets. FEMS Microbiol. Ecol. 83: 504-514.

Zhang T., M.F. Shao and L. Ye. 2012. 454 Pyrosequencing reveals bacterial diversity of activated sludge from 14 sewage treatment plants. ISME, J. 6: 1137-1147.

Zhou M., E. Hernandez-Sanabria and L.L. Guan. 2009. Assessment of the microbial ecology of ruminal methanogens in cattle with different feed efficiencies. Appl. Environ. Microbiol. 75: 6524-6533. 